\title{
Recent Results from the NA49 experiment
}

\section{Christoph Blume for the NA49 Collaboration*}

University of Frankfurt, Germany

E-mail: blumedikf.uni-frankfurt.de

Recent results of the NA49 collaboration are presented. Transverse mass spectra as well as total multiplicities of identified particles are discussed. The study of their evolution from AGS over SPS to the highest RHIC energy reveals a couple of interesting features. These include a sudden change in the energy dependence of the $m_{\mathrm{t}}$-spectra and of the yields of strange hadrons around $30 A \mathrm{GeV}$. Additionally, new results on particle production at high- $p_{\mathrm{t}}$ for $\mathrm{Pb}+\mathrm{Pb}$ collsions at $158 \mathrm{~A} \mathrm{GeV}$, as well as on the $v_{2}$ of $\Lambda$, are discussed.

International Europhysics Conference on High Energy Physics

July 21st - 27th 2005

Lisboa, Portugal

${ }^{*}$ Speaker. 

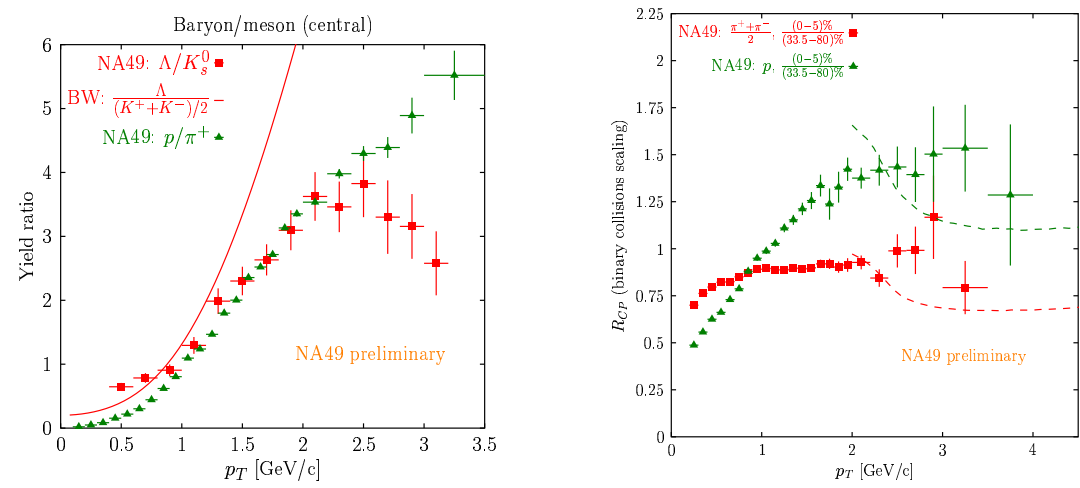

Figure 1: Left: The baryon/meson ratio as a funtion of $p_{\mathrm{t}}$ in central $\mathrm{Pb}+\mathrm{Pb}$ reactions at $\sqrt{s_{\mathrm{NN}}}=17.2 \mathrm{GeV}$. The solid line represents the expectation from a fit with a Blast-Wave model [4]. Right: The nuclear modification factor $R_{\mathrm{CP}}$ as a function of $p_{\mathrm{t}}$ compared to a pQCD calculation including jet quenching (dashed lines).

\section{Introduction}

In the recent years the NA49 experiment has collected data on $\mathrm{Pb}+\mathrm{Pb}$ collisions at beam energies between $20 \mathrm{~A}$ and $158 \mathrm{~A} \mathrm{GeV}$ with the objective to cover the critical region of energy densities where the expected phase transition to a deconfi ned phase might occur in the early stage of the reactions. NA49 is a fi xed target experiment at the CERN SPS. Details on the experimental setup can be found in [1].

\section{High- $p_{\mathrm{t}}$ Spectra}

Among the most interesting features discovered at RHIC is the suppression of high- $p_{\mathrm{t}}$ particle production in central nucleus-nucleus collisions relative to peripheral ones or $\mathrm{p}+\mathrm{p}$ reactions. This suppression can be expressed by the ratio $R_{\mathrm{CP}}=\frac{N_{\text {coll }}(\text { per.) }}{N_{\text {coll }}(\text { cen.) })}$ Yield(cen.) $)$ Yield(per.) As shown in the right panel of Fig. $1, R_{\mathrm{CP}}$ for charged pions always stays below unity for top SPS energies, even though a strong Cronin enhancement should be expected [2]. The data points for pions, as well as for kaons approach at higher $p_{\mathrm{t}}$ a pQCD prediction that includes jet quenching [3], indicating that this effect may already be present at top SPS energies.

The baryon/meson ratio clearly rises above unity for $p_{\mathrm{t}}>1.0 \mathrm{GeV} / c$ (see left panel of Fig. 1), similar as observed at the much higher RHIC energies. This might also point to a possible explanation in terms of quark coalescence models, as are discussed for higher energies.

\section{3. $\Lambda$-Flow}

Figure 2 shows the first results on elliptic flow of $\Lambda$ from NA49. While the p-dependence is similar, the absolute values of $v_{2}$ for $\Lambda$ are always below the ones for protons. This mass ordering is expected from hydrodynamic models, as illustrated by the blast wave fi t [4]. The increase of $v_{2}$ with $p_{\mathrm{t}}$ is more pronounced at higher $\sqrt{s_{\mathrm{NN}}}$ (see right hand side of Fig. 2), which is only partially due to the different centrality selection. 

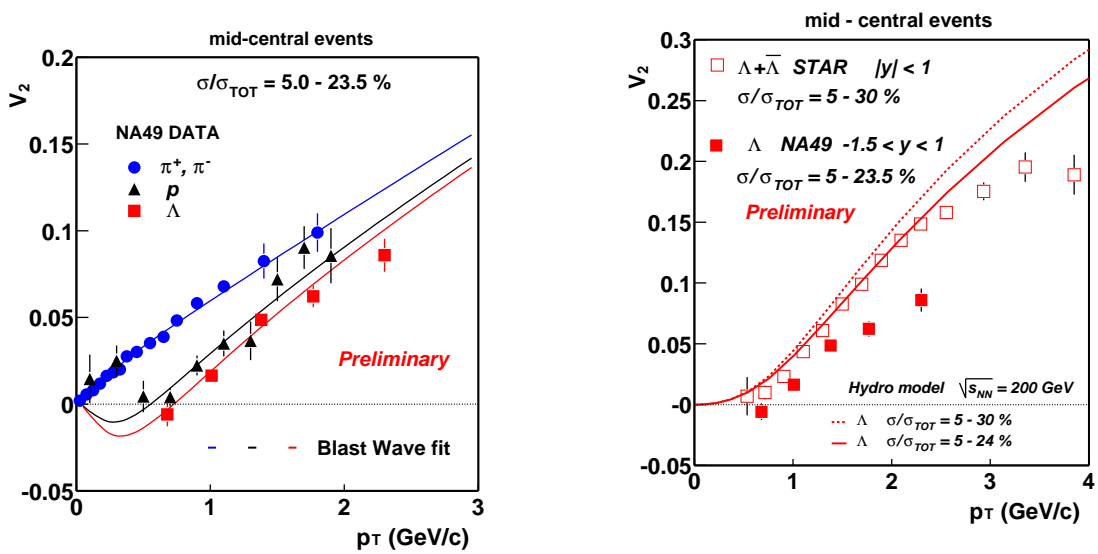

Figure 2: Left: The $v_{2}$ of charged pions, protons, and $\Lambda$ as a function of $p_{\mathrm{t}}$ in semi-central $\mathrm{Pb}+\mathrm{Pb}$ collisions at $\sqrt{s_{\mathrm{NN}}}=17.2 \mathrm{GeV}$. The solid lines represent results of a blast wave fit [4]. Right: The $v_{2}$ for $\Lambda$ measured at $\sqrt{s_{\mathrm{NN}}}=17.2 \mathrm{GeV}$ and $\sqrt{s_{\mathrm{NN}}}=200 \mathrm{GeV}$ [5] in comparison to a hydrodynamical calculation [6].

\section{Transverse mass spectra}

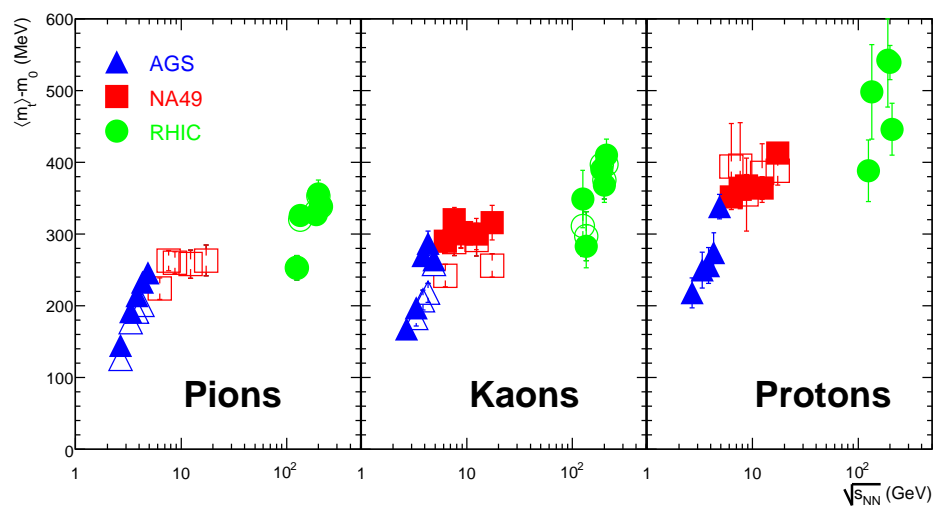

Figure 3: The energy dependence of $\left\langle m_{\mathrm{t}}\right\rangle-m_{0}$ for pions, kaons, and protons at mid-rapidity for 5 (10\%) most central $\mathrm{Pb}+\mathrm{Pb} / \mathrm{Au}+\mathrm{Au}$ reactions. Open (solid) symbols represent negatively (positively) charged particles.

The increase with energy of the inverse slope parameter $T$ of the kaon $m_{\mathrm{t}}$-spectra, as derived from an exponential fi t, exhibits a sharp change to a plateau around $30 \mathrm{~A} \mathrm{GeV} \mathrm{[7].} \mathrm{Since} \mathrm{the}$ kaon $m_{\mathrm{t}}$-spectra - in contrast to the ones of the lighter pions or the heavier protons - have to a good approximation an exponential shape, the inverse slope parameter provides in this case a good characterization of the spectra. For other particle species, however, the local slope of the spectra depends on $m_{\mathrm{t}}$. Instead, the fi rst moment of the $m_{\mathrm{t}}$-spectra can be used to study their energy dependence. The dependence of $\left\langle m_{\mathrm{t}}\right\rangle-m_{0}$ on the center of mass energy $\sqrt{s_{\mathrm{NN}}}$ is summarized in Fig. 3. The change of the energy dependence around a beam energy of $20-30 A \mathrm{GeV}$ is clearly visible for 

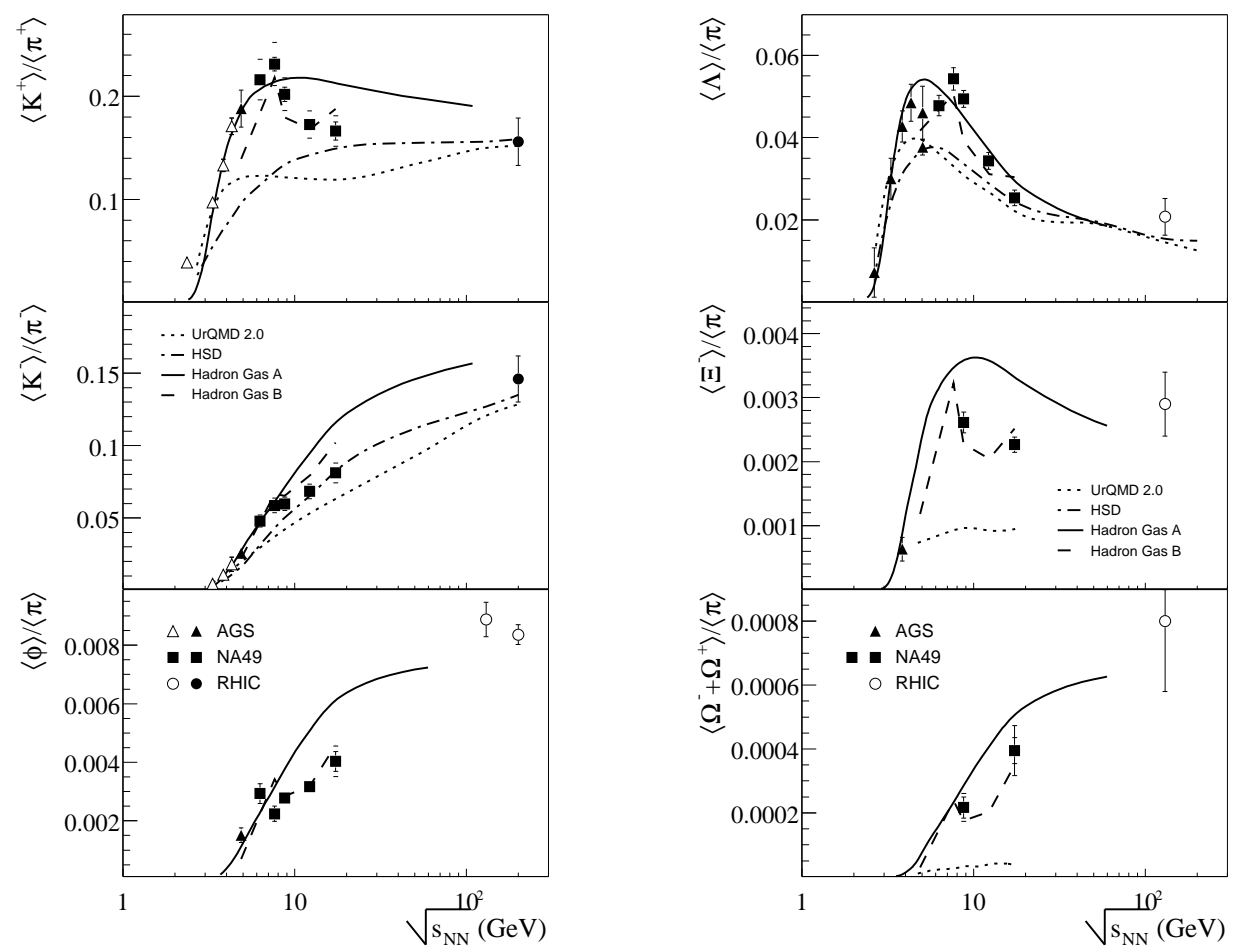

Figure 4: The energy dependence of the $4 \pi$-yields of strange hadrons, normalized to the pion yields, in central $\mathrm{Pb}+\mathrm{Pb} / \mathrm{Au}+\mathrm{Au}$ collisions. The data are compared to string hadronic models $[8,9]$ (UrQMD 2.0: dotted lines, HSD: dashed-dotted lines) and statistical hadron gas models [10,11] (with strangeness undersaturation: dashed line, assuming full equilibrium: solid line).

pions and kaons. While $\left\langle m_{\mathrm{t}}\right\rangle-m_{0}$ rises steeply in the AGS energy range, the rise is much weaker from the low SPS energies on. To a lesser extent this change is also seen for protons.

\section{Particle yields}

In Fig. 4 the energy dependence of the total multiplicities for a variety of strange hadrons, normalized to the pion yield, is summarized and compared to model predictions. Generally, it can be stated that the string hadronic models UrQMD and HSD $[8,9]$ do not provide a good description of the data points. Especially the $\Xi$ and $\Omega$ production is substantially underestimated and the maximum in the $\mathrm{K}^{+} / \pi^{+}$ratio is not reproduced. The statistical hadron gas models [10,11], on the other hand, provide a better overall description of the measurements. However, the introduction of an energy dependent strangeness under-saturation factor $\gamma_{\mathrm{S}}$ is needed [11], in order to capture the structures in the energy dependence of most particle species $\left(\mathrm{K}^{+}, \mathrm{K}^{-}, \phi, \Xi\right)$. This is seen in particular in the energy dependence of the total strangeness production (see Fig. 5), which changes around $30 \mathrm{~A} \mathrm{GeV}$. The strangeness to pion ratio exhibits a pronounced maximum at this energy, which is not reproduced by the statistical hadron gas model [10], but may be related to the onset of deconfi nement [13]. 

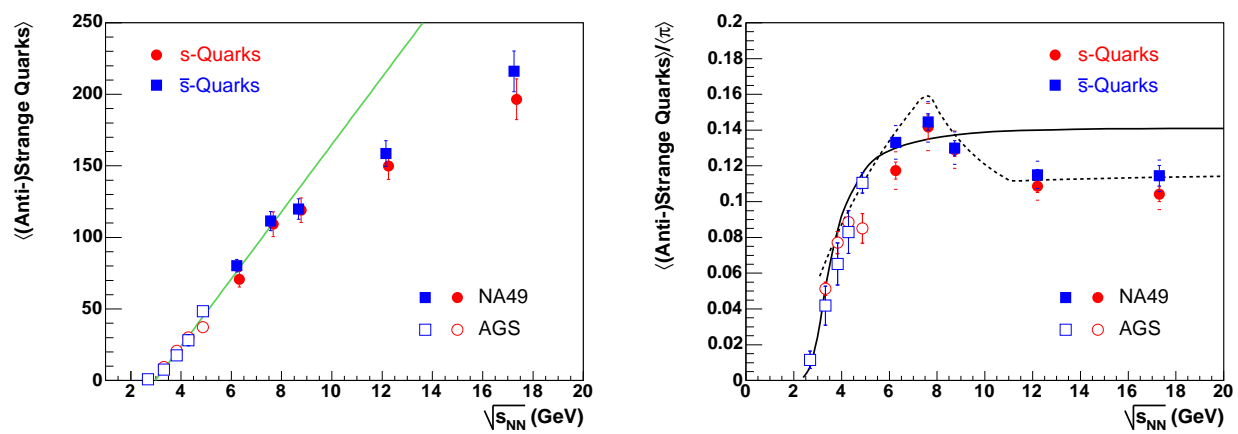

Figure 5: Left: The total number of strange quarks and anti-quarks as carried by kaons and hyperons versus the collision energy for central $\mathrm{Pb}+\mathrm{Pb}(\mathrm{Au}+\mathrm{Au})$ reactions (details see [12]). The line represents a linear fit to the low energy data. Right: The ratio of the number of strange quarks and anti-quarks to the pion yield as a function of $\sqrt{s_{\mathrm{NN}}}$. The solid line represents the statistical hadron gas model with full equilibration of strangeness [10], while the dashed line corresponds to the statistical model of the early stage [13].

\section{References}

[1] S.V. Afanasiev et al. (NA49 collaboration), Nucl. Instrum. Meth. A 430 (1999), 210.

[2] A. Laszlo et al. (for the NA49 collaboration), arXiv:nucl-ex/0510054.

[3] X.N. Wang, Phys. Lett. B 595 (2004), 165.

[4] F. Retiere and M.A. Lisa, Phys. Rev. C 70 (2004), 044907.

[5] J. Adams et al. (STAR collaboration), Phys. Rev. Lett. 92 (2004), 052302.

[6] P. Huovinen, Nucl. Phys. A 761 (2005), 296, and private communication.

[7] M. Gaździcki (for the NA49 collaboration), J. Phys. G 30 (2004), S701.

[8] M. Bleicher et al., J. Phys. G 25 (1999), 1859.

[9] E.L. Bratkovskaya et al., Phys. Rev. C 69 (2004), 054907.

[10] P. Braun-Munziger, J. Cleymans, H. Oeschler, and K. Redlich, Nucl. Phys. A 697 (2002), 902.

[11] F. Becattini, M. Gaździcki, A. Keränen, J. Manninen, and R. Stock, Phys. Rev. C 69 (2004), 024905.

[12] C. Blume et al. (for the NA49 collaboration), J. Phys. G 31 (2005), s685.

[13] M. Gaździcki and M.I. Gorenstein, Acta Phys. Polon. B 30 (1999), 2705. 